\title{
Can coronal hole spicules reach coronal temperatures?`
}

\author{
M. S. Madjarska, K. Vanninathan, and J. G. Doyle
}

\begin{abstract}
Armagh Observatory, College Hill, Armagh BT61 9DG, N. Ireland, UK
e-mail: madj@arm.ac.uk
\end{abstract}

Received 16 February 2011 / Accepted 11 May 2011

\section{ABSTRACT}

\begin{abstract}
Aims. The present study aims to provide observational evidence of whether coronal hole spicules reach coronal temperatures. Methods. We combine multi-instrument co-observations obtained with the SUMER/SoHO and with the EIS/SOT/XRT/Hinode. Results. The analysed three large spicules were found to be comprised of numerous thin spicules that rise, rotate, and descend simultaneously forming a bush-like feature. Their rotation resembles the untwisting of a large flux rope. They show velocities ranging from 50 to $250 \mathrm{~km} \mathrm{~s}^{-1}$. We clearly associated the red- and blue-shifted emissions in transition region lines not only with rotating but also with rising and descending plasmas. Our main result is that these spicules although very large and dynamic, are not present in the spectral lines formed at temperatures above $300000 \mathrm{~K}$.

Conclusions. In this paper we present the analysis of three Ca II H large spicules that are composed of numerous dynamic thin spicules but appear as macrospicules in lower resolution EUV images. We found no coronal counterpart of these and smaller spicules. We believe that the identification of phenomena that have very different origins as macrospicules is due to the interpretation of the transition region emission, and especially the He II emission, wherein both chromospheric large spicules and coronal X-ray jets are present. We suggest that the recent observation of spicules in the coronal AIA/SDO $171 \AA$ and $211 \AA$ channels probably comes from the existence of transition region emission there.
\end{abstract}

Key words. Sun: corona - Sun: transition region - line: profiles - methods: observational

\section{Introduction}

The term spicule refers to jet-like features expelled from the chromosphere as seen at the solar limb. They were first observed by Secchi (1877) and called "spicules" by Roberts (1945). Spicules are best viewed at the solar limb as bright features against the dark background of the solar corona in $\mathrm{H} \alpha$ and $\mathrm{Ca}$ II images. Several studies report that these phenomena fall back along the same trajectory or fade out (Beckers 1972; Suematsu 1998). Many on-disk filament-like features were identified as the counterpart of the limb spicules due to the similarities in their properties (Christopoulou et al. 2001; Rouppe van der Voort et al. 2007), and some were named "mottles" (Tsiropoula \& Schmieder 1997). Coronal hole spicules are found to be taller than quiet Sun spicules, probably owing to the different configuration of the magnetic field of the two regions (Beckers 1972). Spicules/mottles have been observed at temperatures between $5000 \mathrm{~K}$ and $300000 \mathrm{~K}$. However, De Pontieu et al. (2011) report that a small but sufficient fraction of spicules, including coronal hole spicules, are heated to temperatures above $1 \mathrm{MK}$ based on observations from the Atmospheric Imaging Assembly (AIA) instrument onboard the Solar Dynamic Observatory (SDO) taken with the $171 \AA$ filter. Transient events like spicules/mottles are of prime importance as they intermittently connect the chromosphere with the corona and possibly sustain the mass balance in the solar atmosphere (Tsiropoula \& Tziotziou 2004). However, the downflow observed in transition region lines may be the result of the mottle/spicule plasma returning to the solar surface (Pneuman \& Kopp 1978; Withbroe 1983; Tsiropoula $\&$ Tziotziou 2004). If all the material that is sent up through spicules/mottles is returned to the solar surface, then their contribution to coronal heating will be minimal, dismissing the

* Movie is available in electronic form at http://www . aanda.org possibility of spicules directly contributing to coronal heating (Withbroe 1983). Moreover, there are speculations that spicules may be capable of transporting energy high into the upper chromosphere and even up to the corona (Pneuman \& Kopp 1978; Athay \& Holzer 1982) so they can be possibly responsible for coronal heating (Athay 2000; De Pontieu et al. 2011).

Bohlin et al. (1975) revealed the existence of jet-like features found in images taken with the slitless NRL spectrograph during the Skylab mission. The events were called macrospicules because they resembled $\mathrm{H} \alpha$ spicules, but were much larger and had longer lifetimes. They appear increasingly inclined away from the pole as a function of increasing position angle measured from the pole, which makes them comparable to $\mathrm{H} \alpha$ spicules. They were first seen in He II $304 \AA$ and only rarely in Ne VII $465 \AA$ $(5.7 \mathrm{~K})$ (Bohlin et al. 1975). We would like to note here that the formation temperature of He II ranges from 5 to $12 \times 10^{4} \mathrm{~K}$ with a maximum at $8 \times 10^{4} \mathrm{~K}$, corresponding to a low transition region temperature or the uppermost chromosphere (Jordan 1974). Because of the anomalous behaviour of the He I and II lines (Andretta et al. 2003), observation of transient events in these lines does not tell where in the solar atmosphere these events originate. Therefore, to link chromospheric features, such as spicules, with phenomena seen at temperatures describing the transition region and corona, is not a trivial task and requires the use of suitable data.

Spectral and imaging macrospicule analysis were reported in several papers (Pike \& Harrison 1997; Parenti et al. 2002; Pike \& Mason 2002; Kamio et al. 2010). In SoHO/CDS data, they were registered in spectral lines with formation temperatures from $20000 \mathrm{~K}$ to $1 \mathrm{MK}$ (Pike \& Harrison 1997), although Pike \& Mason (2002) find no Mg IX (1 MK) emission in the region where macrospicules were detected in He I $(20000 \mathrm{~K})$ and $\mathrm{O}$ V (250000 K). Recently, Kamio et al. (2010) have 

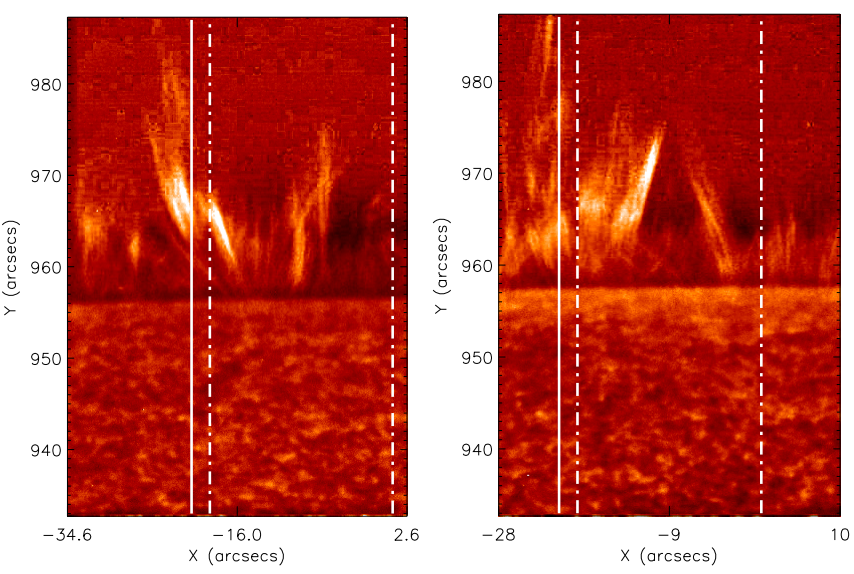

described a macrospicule seen in the He II $256 \AA$ and Fe XII $195 \AA(1.2 \mathrm{MK})$ lines from the Extreme-ultraviolet Imaging Spectrometer (EIS) onboard the Hinode satellite. The authors associated the macrospicule with an X-ray jet from a coronal bright point but no counterpart was reported in SOT Ca II H. Sterling et al. (2010) present observations of an X-ray jet in X-ray telescope (XRT) images that are also seen in EIS 266" slot images consisting of a blend of several lines including He II and Fe XV. The X-ray jet was associated with tall spicules seen in images from the Solar Optical Telescope (SOT) taken with the Ca II H filter and identified as type II spicules.

We performed specially planned multi-wavelength Hinode (EIS/SOT/XRT) and SoHO (Solar Measurements of Emitted Radiation (SUMER)) co-observations at the solar limb. The present paper describes the association of simultaneously evolving spicules seen in SOT Ca II with macrospicules, judging from their appearance in SUMER and EIS observations. The aim of our investigation was to probe the possibility that spicules reach coronal temperatures, i.e. can deposit thermal energy directly in the solar corona. Section 2 describes the analysed data and their alignment. In Sect. 3 we give the obtained results. In Sect. 4 we discuss the open questions and state our conclusions.

\section{Observations}

The observations were taken at the North Pole on 2009 April 28 and 29. The events were registered by the SOT, EIS, and XRT onboard Hinode and the SUMER spectrometer onboard SoHO (Fig. 1). The SOT (Tsuneta et al. 2008) took observations with the $\mathrm{Ca}$ II $\mathrm{H}$ filter with a cadence of $10 \mathrm{~s}$. The EIS (Culhane et al. 2007) observations were done with a $2^{\prime \prime}$ slit and a 60 s exposure time. They consist of a large raster with a size of $70^{\prime \prime} \times 248^{\prime \prime}$ followed by small rasters of $24^{\prime \prime} \times 248^{\prime \prime}$. EIS took observations in many spectral lines, e.g. Fe VIII, Fe X, Fe XI up to Fe XXIII, $\mathrm{O} \mathrm{V}$ and VI, and He II. We discuss only the strongest ones, i.e. He II $256.32 \AA$ (4.7 K) and Fe XII $195.12 \AA$ (6.1 K). The XRT (Golub et al. 2007) was observing with the Al poly filter in a field-of-view (FOV) of $384^{\prime \prime} \times 384^{\prime \prime}$ with a cadence of $30 \mathrm{~s}$. The SUMER spectrometer (Wilhelm et al. 1995) took a large raster followed by sit-and-stare observations using the $1^{\prime \prime} \times 300^{\prime \prime}$ slit in O v $629.77 \AA$, N v $1238.82 \AA$, Mg X $624.90 \AA$ and several chromospheric lines (C I, Si II, and S II). The O V $629 \AA$ line was used to obtain relative Doppler shift maps with the rest wavelength taken as an average from the whole dataset.

Aligning data from various instruments was based on images obtained in spectral lines or filters with similar temperatures. First, a SUMER Mg X sit-and-stare image was aligned with a co-temporal slice-time image from XRT. SUMER and SOT images were then aligned. Approximately nine pixels from an SOT

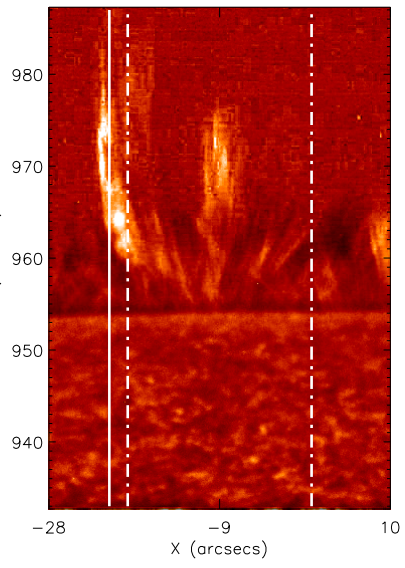

Fig. 1. SOT Ca II images showing spicules taken from left to right on 2009 April 28 at 16:24 UT, on April 29 at 02:24 UT and 03:28 UT. The overplotted solid line indicates the position of the SUMER slit, while the dasheddotted lines indicate the EIS raster FOV.

image correspond to $1^{\prime \prime}$, which is equivalent to the SUMER slit width. An automated procedure was written to cut a slice of 9 pixels in time from the SOT images and compare them with a SUMER sit-and-stare image in the Si II, N v, and O V lines. Due to the uncertainties of the instrument pointing, it is necessary to search for the real position using an observed region of at least $\pm 20^{\prime \prime}$ from the commanded coordinates. We were satisfied with our alignment only when all off-limb features seen in the SUMER observations were identified with their SOT Ca II H counterpart. The EIS data were easily aligned with XRT using EIS images taken in high-temperature lines. The alignment was done (Fig. 1) with a precision of $\pm 2^{\prime \prime}$ to $\pm 4^{\prime \prime}$, which is within reasonable limits for the size of the observed events (more than $25^{\prime \prime}$ in Solar X).

\section{Results}

The observed regions are seeded with thin dynamic jet-like events that correspond to the type II spicules (De Pontieu et al. 2007, 2011) observed in coronal holes (see Fig. A.1). After a very challenging but successful alignment of all instrument data, we selected the three largest events that fall under the SUMER slit for further analysis. They are comprised of numerous highvelocity, thin, long spicules (denoted type II spicules), which evolve simultaneously. The reason for analysing only the largest spicules is the lower spatial resolution of SUMER, EIS and XRT compared to SOT (for more details see Sect. 2). The ratio of the spatial resolution of EIS ( $2^{\prime \prime}$ slit width) to SOT $\left(0.1089^{\prime \prime}\right)$ is approximately 18 , which means that a feature identified in SOT has to be larger by more than twice the spatial resolution of EIS, i.e. more than $4^{\prime \prime}$ in width, in order to be identified in EIS data. In Fig. 1 we show the three spicules that represent "bushes" of many thin spicules, which rise simultaneously forming a large spicule (see Fig. A.1). They reach various heights (in average more than $20000 \mathrm{~km}$ ) above the solar limb. Their bush-like horizontal expansion reaches up to $25000 \mathrm{~km}$. The proper motion of the spicules was estimated by following individual spicules in as many images as possible. We found a speed of rising between 50 and $250 \mathrm{~km} \mathrm{~s}^{-1}$. The descending velocity was difficult to estimate because of the superposition of rotation, up and downflows. We found a persistent blue-shifted emission of less than $50 \mathrm{~km} \mathrm{~s}^{-1}$ even when rotation is not present which apparently corresponds to a down-flow.

The event on April 28 happened at around 16:25 UT and had a duration of $10 \mathrm{~min}$ in SOT Ca II. It was registered in SUMER O V 2 min later, i.e. 16:27 UT, and lasted around $12 \mathrm{~min}$. The phenomenon can be described as multiple thin jets that are seen to shoot upwards at high speed. They rotate as one unit while rising and finally disappear into the surroundings. The first event 
of April 29 took place at around 02:25 UT. The jet-like event rose almost from a blast and expended beyond the SOT FOV, i.e. more than $40000 \mathrm{~km}$ above the solar limb. Just like the previous event, it also rotated and then disappeared. The third spicule rose quickly at around 03:21 UT and sustained its height, rotating for a longer period than the previously discussed events before it descended. Again, we observed a delay in the appearance of the event in the SUMER O V line. The duration of both events on April 29 is approximately $10 \mathrm{~min}$.

We analysed the SUMER data taken in the chromospheric Si II $1250.41 \AA$ blended with the C I $1250.42 \AA$ and S II $1250.58 \AA$ lines, the transition region O V $629.76 \AA$ and $\mathrm{N} \mathrm{V} 1238.82 \AA$ lines, and the coronal Mg X $624.9 \AA$ line. The spicules are clearly present in the chromospheric lines. In these lines, as well as in transition region lines, the spicules appear to be the so-called "macrospicules", i.e. larger in size than the $\mathrm{Ca}$ II $\mathrm{H}$ spicules. In addition to the largest spicules discussed here, one can see several smaller SUMER spicules that are also composed of several thin, long SOT Ca II spicules. The features can be seen evolving under the SUMER slit in the edge enhanced image movie (Fig. A.1). In the transition region lines, the spicules show strong blue- and red-shifted emission (Fig. 2). For the first time we were able to establish the meaning of the blue and red-shifted emission at this line-of-sight position. From the comparison of the simultaneously taken SUMER and SOT data (Fig. 2), we found that the Doppler shifts that were interpreted earlier by Cook et al. (1984), Pike \& Harrison (1997), and Parenti et al. (2002) as rotating motions, do indeed indicate a rotation, but they also mean an upflow that corresponds to a redshifted emission and a blueshift that is consistent with downflow. The blueshift was persistent once the event takes off. We should note that a single Gauss fit as presented in Fig. 2 does not really give a full picture of the dynamics of these events. Only a careful analysis of individual pixels with strong enough signal provides the true evolution of the spicule plasmas.

The next step in our study was the analysis of spectral lines formed at coronal temperatures. No signal was detected in the coronal SUMER Mg X line for the duration of the large spicules. However, due to the uncertainties of using this line for coronal diagnostics (Madjarska 2011), we omit this observation from our conclusions. We concentrated our analysis on the behaviour of the EIS spectral lines, which are perfectly suitable for coronal diagnostics. In order to identify the spicules in EIS data, we used the He II $256.32 \AA$ line as reference. In Fig. 3 we present a raster taken at 15:28 UT (the last good raster before the large spicules took off) on April 28 with no visible off-limb features. By taking a limb contour in the He II $256 \AA$, we established our reference line which will represent the solar limb. The presence of the spicule in the He II raster image (the EIS FOV is shown in Fig. 1), is more than evident. The large spicule is also seen in the $\mathrm{O} v$ line, but the low signal in this line does not permit to show a representative image. The analysis of all iron lines from Fe VIII up to Fe XVI shows no evidence of a spicule at their corresponding formation temperatures. In Fig. 3 (third and fourth images), we give example raster images in the strongest line in the EIS spectrum, the Fe XII $195.12 \AA$ line, before (third) and during (fourth) the course of the SUMER spicule. The jet-like features seen in the Fe XII raster images correspond to a X-ray jet from a bright point that is situated below the limb. This has been established by using an XRT animated sequence. The same is shown in Fig. 4 for the two events on April 29. Again, no trace of the large spicule is seen in the coronal lines.
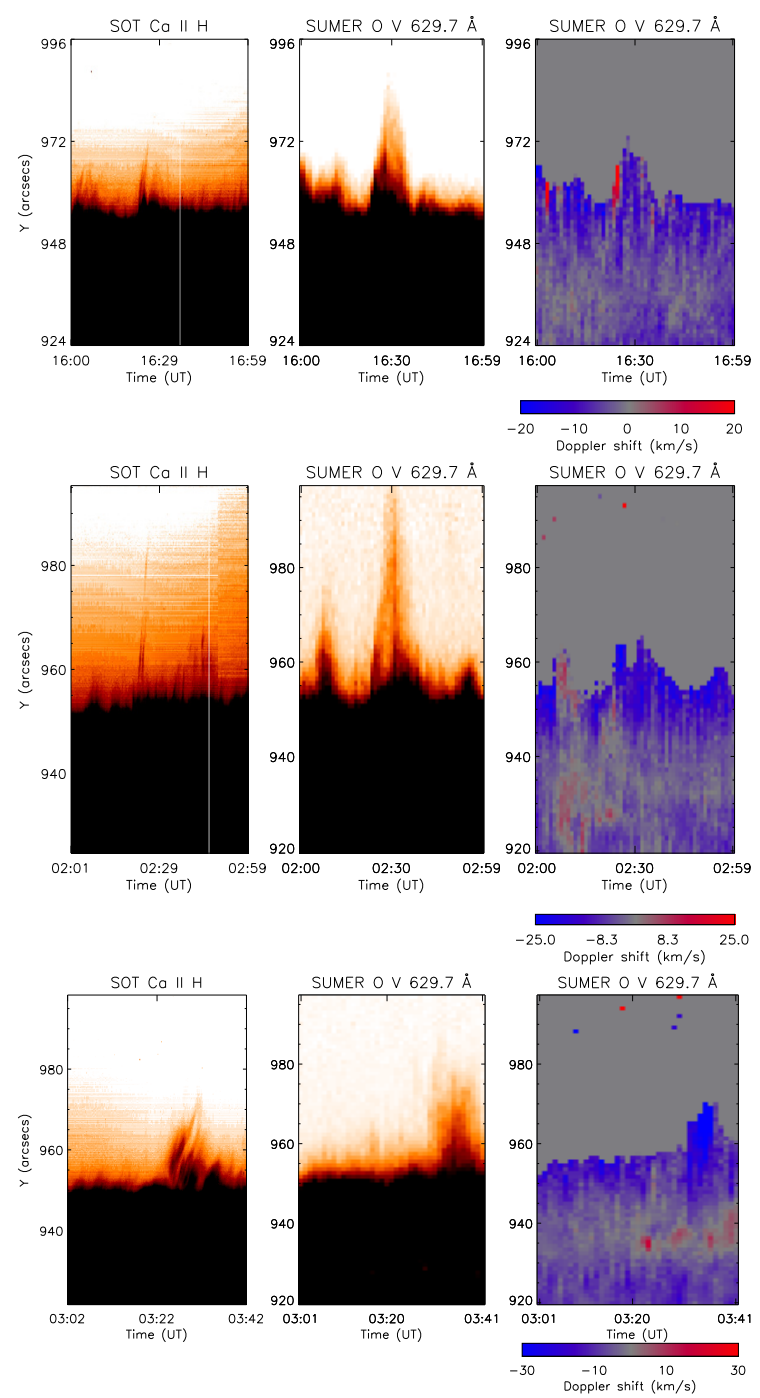

Fig. 2. Left to right: SOT Ca II H slice-time images, the corresponding SUMER sit-and-stare radiance and Doppler velocity $\mathrm{O} \mathrm{v}$ images on April 28 (top) and April 29 (middle and bottom).
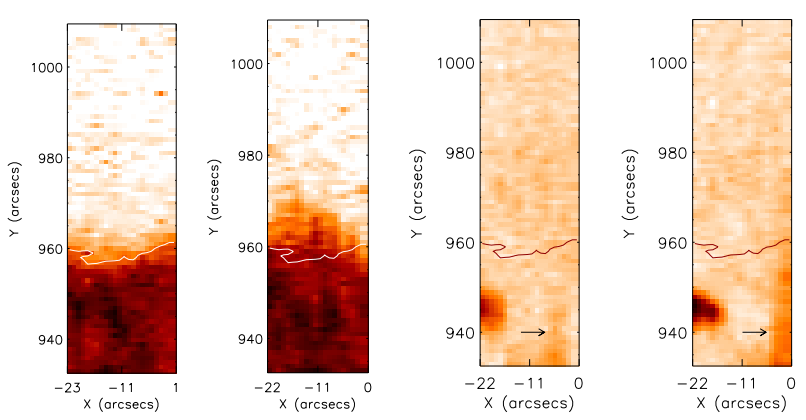

Fig. 3. EIS rasters taken on April 28 starting at 15:28 UT and 16:23 UT in the He II $256 \AA$ A line (first and second images) and the Fe XII $195 \AA$ line (third and fourth images). The arrows indicate the X-ray jet from a coronal bright point identified from the XRT images.

\section{Discussion and conclusions}

In this paper we present the analysis of three large spicules called macrospicules as seen in EUV spectral lines. The macrospicules appear to consist of many thread-like spicules as seen in highresolution SOT Ca II H images, which rise, rotate, descend, and in general evolve almost simultaneously. We identified the counterpart of these large spicules in SUMER sit-and-stare and EIS raster images. The large SOT spicules are very dynamic and 
appear to be features which are usually identified as macrospicules in transition region emission lines. Our main result is that although very large and dynamic, these spicules do not appear in spectral lines formed at temperatures above $300000 \mathrm{~K}$. Macrospicules have been reported in coronal lines (Pike \& Harrison 1997), and recently a feature called macrospicule identified in the EIS He II line was clearly seen as an X-ray jet (Kamio et al. 2010).

Where does the discrepancy between our results and these observations comes from? We strongly believe that the misidentification, or rather the identification, of a different type of phenomena as macrospicules comes from the interpretation of the transition region emission and, especially, the He I and II emission. Our recent multi-instrument analysis of a "classical" X-ray jet has clearly demonstrated that X-ray jets are strongly visible in the He II emission (Madjarska 2011). We believe that this is also the case of the event described by Kamio et al. (2010). Equally, spicules are prominent in these spectral lines. Forthcoming papers by Madjarska et al. (in prep.) and Subramanian et al. (in prep.) will give more information on this subject.

De Pontieu et al. (2011) reports observation of spicules in the AIA/SDO $171 \AA$ channel in active regions and the quiet Sun as well as in coronal holes (Fig. 3 in their paper and movies S8 and S9). This made us ask why we do not detect any coronal emission in EIS data of coronal hole spicules. It should be noted here that these two works deal with different types of observations, both spectroscopic and imaging, so we decided to make a preliminary spectroscopic analysis of the AIA $171 \AA$ channel. This channel is dominated by Fe IX emission (http://aia.lmsal.com/public/results.htm). However, it also contains an abundance of cool Fe VIII, Ne V, O V, and VI lines and therefore, when along the line-of-sight there is no emission from the Fe IX lines, the cooler lines will have a significant contribution. This was clearly demonstrated from simultaneous TRACE Fe IX/X $171 \AA$, and SUMER transition region and coronal observations (Madjarska \& Doyle 2008, see Sect. 4). In order to make a preliminary analysis of the cooler emission contribution in the AIA 171, we checked co-observations of a large prominence by EIS and AIA. Solar prominences have very similar plasma parameters to spicules concerning temperatures and densities, but the prominences are much larger, especially quiescent ones. Surprisingly, we clearly identified all solar prominences in the AIA $171 \AA$ images. A check on the EIS observations for one of these prominences revealed an emission that is not higher than Fe VIII with a contribution function peaking at $400000 \mathrm{~K}$, i.e. at transition region temperature. Is it, therefore, possible that the spicules seen by De Pontieu et al. (2011) in the AIA $171 \AA$ channel come from the emission of lower temperature lines? This question needs to be answered by careful spectroscopic studies of the response of the AIA channels.

\section{Appendix A: Still figure from movie}

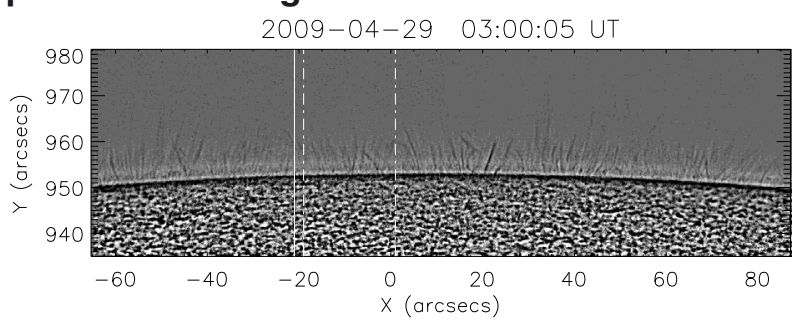

Fig. A.1. SOT Ca II movie of spicules in coronal hole region on April 29. The over-plotted solid line indicates the position of the SUMER slit and the dashed-dotted lines indicate the left and right edges of the EIS FOV rasters.

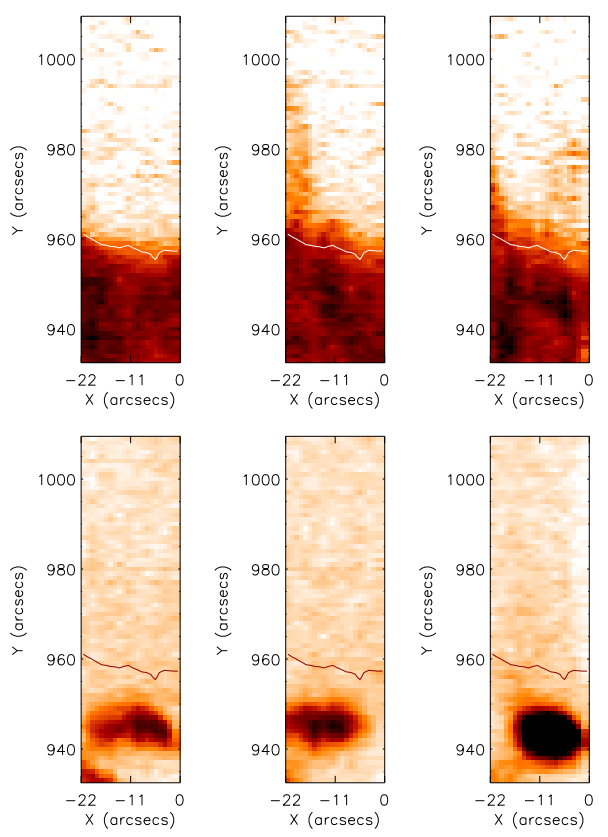

Fig. 4. EIS rasters taken on April 29 at 02:09 UT (left), 02:19 UT (middle) and 03:23 UT (right). The top and bottom row images were taken in the He II $256 \AA$ and Fe XII $195 \AA ̊$ Aines, respectively.

Acknowledgements. The authors thank ISSI for the support of the team "Smallscale transient phenomena and their contribution to coronal heating". Research at Armagh Observatory is grant-aided by the N. Ireland Department of Culture, Arts and Leisure. We also thank STFC for support via grants ST/F001843/1 and ST/H001921/1. Hinode is a Japanese mission developed and launched by ISAS/JAXA, with NAOJ as domestic partner and NASA and STFC (UK) as international partners.

\section{References}

Andretta, V., Del Zanna, G., \& Jordan, S. D. 2003, A\&A, 400, 737

Athay, R. G. 2000, Sol. Phys., 197, 31

Athay, R. G., \& Holzer, T. E. 1982, ApJ, 255, 743

Beckers, J. M. 1972, ARA\&A, 10, 73

Bohlin, J. D., Vogel, S. N., Purcell, J. D., et al. 1975, ApJ, 197, L133

Christopoulou, E. B., Georgakilas, A. A., \& Koutchmy, S. 2001, Sol. Phys., 199, 61

Cook, J. W., Brueckner, G. E., Bartoe, J., \& Socker, D. G. 1984, Adv. Space Res., 4, 59

Culhane, J. L., Harra, L. K., James, A. M., et al. 2007, Sol. Phys., 243, 19

De Pontieu, B., McIntosh, S., Hansteen, V. H., et al. 2007, PASJ, 59, 655

De Pontieu, B., McIntosh, S. W., Carlsson, M., et al. 2011, Science, 331, 55

Golub, L., Deluca, E., Austin, G., et al. 2007, Sol. Phys., 243, 63

Jordan, C. 1974, A\&A, 34, 69

Kamio, S., Curdt, W., Teriaca, L., Inhester, B., \& Solanki, S. K. 2010, A\&A, 510, L1

Madjarska, M. S. 2011, A\&A, 526, A19

Madjarska, M. S., \& Doyle, J. G. 2008, A\&A, 482, 273

Parenti, S., Bromage, B. J. I., \& Bromage, G. E. 2002, A\&A, 384, 303

Pike, C. D., \& Harrison, R. A. 1997, Sol. Phys., 175, 457

Pike, C. D., \& Mason, H. E. 2002, Sol. Phys., 206, 359

Pneuman, G. W., \& Kopp, R. A. 1978, Sol. Phys., 57, 49

Roberts, W. O. 1945, ApJ, 101, 136

Rouppe van der Voort, L. H. M., De Pontieu, B., Hansteen, V. H., Carlsson, M., \& van Noort, M. 2007, ApJ, 660, L169

Secchi, P. A. 1877, Le Soleil, 2

Sterling, A. C., Harra, L. K., \& Moore, R. L. 2010, ApJ, 722, 1644

Suematsu, Y. 1998, in Solar Jets and Coronal Plumes, ed. T.-D. Guyenne, ESA SP-421, 19

Tsiropoula, G., \& Schmieder, B. 1997, A\&A, 324, 1183

Tsiropoula, G., \& Tziotziou, K. 2004, A\&A, 424, 279

Tsuneta, S., Ichimoto, K., Katsukawa, Y., et al. 2008, Sol. Phys., 249, 167

Wilhelm, K., Curdt, W., Marsch, E., et al. 1995, Sol. Phys., 162, 189

Withbroe, G. L. 1983, ApJ, 267, 825 